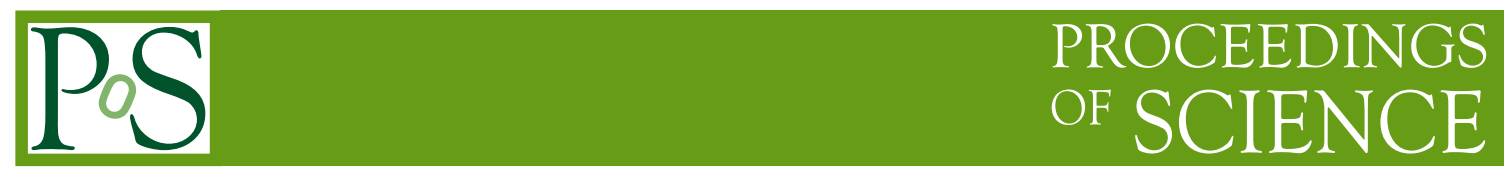

\title{
The Foot (Fragmentation Of Target) Experiment
}

\author{
S. Argiro ${ }^{a, n}$, D. Barbosa ${ }^{b, u}$, G. Battistoni ${ }^{c}$, N. Belcari ${ }^{b, u}$, G. Bruni ${ }^{d}$, M. G. Bisogni ${ }^{b, u}$, \\ S. Brambilla ${ }^{c}$, N. Camarlinghi ${ }^{b, u}$, P. Cerello ${ }^{a}$, E. Ciarrocchi ${ }^{b, u}$, A. Clozza ${ }^{e}$, \\ G. De Lellis ${ }^{f, t}, \mathbf{A}$. Di Crescenzo ${ }^{f, t}$ M. Durante ${ }^{g}$, R. Faccini ${ }^{h, p}$, V. Ferrero ${ }^{a}$, \\ F. Ferroni ${ }^{h, p}$, M. Fioralelli ${ }^{f}$, M. Franchini ${ }^{d, v}$, M. Garbini ${ }^{d, v}$, G. Giraudo ${ }^{a}$ S. Hild ${ }^{g}$, \\ A. lacomino, ${ }^{f}$, A. Lauria ${ }^{f, t}$, C. La Tessa ${ }^{g, r}$ J. Lotti ${ }^{f}$, I. Mattei ${ }^{c}$, M. Marafini ${ }^{h, m}$ \\ M. C. Montesi ${ }^{f, t}$, M. C. Morone ${ }^{i, s}$, M. Morrocchi ${ }^{b, u}$, S. Muraro ${ }^{b}$, L. Narici ${ }^{i, s}$, \\ R. Paramatti ${ }^{h, p}$, N. Pastrone ${ }^{a}$, V. Patera ${ }^{* h, p, m}$, C. Peroni ${ }^{a, n}$, L. Ramello ${ }^{a, n}$, \\ V. Rosso ${ }^{b, u}$, M. Rovituso $g$, C. Sanelli ${ }^{e}$, M. Salvatore ${ }^{l}$, A. Sarti ${ }^{e, p, m}$, G. Sartorelli ${ }^{d, v}$, \\ O. Sato ${ }^{q}$, A. Schiavi ${ }^{h, p}$, E. Scifoni ${ }^{g}$, A. Sciubba ${ }^{h, p, m}$. M. Selvi ${ }^{d}$, L. Servoli ${ }^{l}$, \\ M. Sitta ${ }^{a, o}$, R. Spighi ${ }^{d}$, P. Spinnato ${ }^{g}$, E. Spiriti ${ }^{e}$, G. Sportelli ${ }^{b, u}$, M. Testa ${ }^{e}$, \\ V. Tioukov ${ }^{f}$ F. Tommasino ${ }^{g, r}$, G. Traini ${ }^{h, p}$, S. M. Valle ${ }^{c, z}$, M. Villa ${ }^{d, v}$, A. Zoccoli ${ }^{d, v}$ \\ a INFN, Sezione di Torino, Italy \\ ${ }^{b}$ INFN, Sezione di Pisa, Italy \\ ${ }^{c}$ INFN, Sezione di Milano, Italy \\ ${ }^{d}$ INFN, Sezione di Bologna, Italy \\ ${ }^{e}$ INFN, Laboratori Nazionali di Frascati, Italy \\ ${ }^{f}$ INFN, Sezione di Napoli, Italy \\ ${ }^{g}$ INFN, Trento Institute for Physics Application, Italy \\ ${ }^{h}$ INFN, Sezione di Roma 1, Italy \\ ${ }^{i}$ INFN, Sezione di Roma 2, Italy \\ ${ }^{l}$ INFN, Sezione di Perugia, Italy \\ ${ }^{m}$ Museo Storico della Fisica e Centro Studi e Ricerche Enrico Fermi, Italy \\ ${ }^{n}$ Torino University, Italy \\ ${ }^{o}$ Piemonte Orientale University, Italy \\ ${ }^{p}$ Roma "La Sapienza" University, Italy \\ ${ }^{q}$ Nagoya University, Japan \\ ${ }^{r}$ Trento University, Italy \\ ${ }^{s}$ Roma "Tor Vergata" University, Italy \\ ${ }^{t}$ Napoli "'Federico II" University, Italy \\ "Pisa University, Italy \\ ${ }^{v}$ Bologna University, Italy \\ ${ }^{z}$ Milano University, Italy
}


Particle therapy uses proton or ${ }^{12} \mathrm{C}$ beams for the treatment of deep-seated solid tumors. Due to the features of energy deposition of charged particles a small amount of dose is released to the healthy tissue in the beam entrance region, while the maximum of the dose is released to the tumor at the end of the beam range, in the Bragg peak region. However nuclear interactions between beam and patient tissues induce fragmentation both of projectile and target and must be carefully taken into account.

In ${ }^{12} \mathrm{C}$ treatments the main concern are long range fragments due to projectile fragmentation that release dose in the healthy tissue after the tumor, while in proton treatment the target fragmentation produces low energy, short range fragments along all the beam range.

The FOOT experiment (FragmentatiOn Of Target) is designed to study these processes. Target nuclei $\left({ }^{16} \mathrm{O},{ }^{12} \mathrm{C}\right)$ fragmentation induced by $150-250 \mathrm{AMeV}$ proton beam will be studied via inverse kinematic approach. ${ }^{16} \mathrm{O},{ }^{12} \mathrm{C}$ therapeutic beams, with the quoted kinetic energy, collide on graphite and hydrocarbons target to provide the cross section on Hydrogen. This configuration explores also the projectile fragmentation of these ${ }^{16} \mathrm{O},{ }^{12} \mathrm{C}$ beams. The detector includes a magnetic spectrometer based on silicon pixel detectors and drift chamber, a scintillating crystal calorimeter with TOF capabilities, able to stop the heavier fragments produced, and a $\Delta E$ detector to achieve the needed energy resolution and particle identification.

An alternative setup of the experiment will exploit the emulsion chamber capabilities. A specific emulsion chambers will be coupled with the interaction region of the FOOT setup to measure the production in target fragmentation of light charged fragments as protons, deuterons, tritons and Helium nuclei.

The FOOT data taking is foreseen at the CNAO experimental room and will start during early 2018 with the emulsion setup, while the complete electronic detector will take data since 2019

The 26th International Nuclear Physics Conference

11-16 September, 2016

Adelaide, Australia

${ }^{*}$ Speaker. 


\section{Introduction}

In the last decade a continuous increase in the number of cancer patients treated with Charged Particle Therapy (CPT) [1] has been registered, due to its effectiveness in the treatment of deepseated solid tumors [2]. At the end of 2014 more than 137000 patients received CPT, mostly with protons (86\%) and with carbon ions (11\%). Interest is recently arising in the use of helium and oxygen as therapeutic ions. The depth-dose profile of charged particles is characterized by an entrance channel where a low amount of dose is released, followed by a narrow region, the Bragg Peak (BP), where the maximum of the dose is deposited and that is usually seated on the cancer region, allowing sparing healthy tissues. Furthermore the increase in Linear Energy Transfer (LET) at the end of range produces an enhanced biological effectiveness in cell killing in the BP region compared to conventional photon radiation. This effect is particularly efficient for ${ }^{12} \mathrm{C}$ beam. In biophysics the Relative Biological Effectiveness (RBE), ratio of photon to charged particle dose producing the same biological effect, quantifies this effectiveness. Even though track structure also plays a role, as a general approximation, high LET corresponds to high RBE.

In proton treatment, due to low LET and to high energy of the proton beam in the entrance channel, a RBE close to one would be expected. Actually, a constant RBE equal to 1.1 is currently assigned to protons in treatment planning. However, the experiments show a significant increase in RBE above 1.1 [3] and the topic of RBE variability in protontherapy has been widely debated in recent years. In particular, as shown in ref. [4], the increase of RBE can give rise to a biological range extension after $\mathrm{BP}$ or to an increase of biological damage in the entrance channel (plateau region in the Bragg curve), i.e. in the region of healthy tissues. In fact it emerges that using a constant $\mathrm{RBE}=1.1$ can lead to an under or overestimation of the dose in the healthy tissue region.

An hypothesis proposed in ref. [4] is that particles produced in target fragmentation could be one of the causes contributing to the increase of RBE of protons. When crossing the patient, nuclear interactions occur between the beam and the patient tissues. In the case of proton beams, only target fragmentation occurs, generating a spectrum of low energy heavy recoils that depends on beam energy and on target materials. These secondary charged particles have short range (e.g. order of 10-100 $\mu \mathrm{m}$ ), very high LET and then high RBE. For heavier ion beams, projectile fragmentation also takes place and is responsible for the dose tail observed after the BP.

The process of target fragmentation can have an impact in particular in the plateau region, where is estimated almost $10 \%$ of proton interactions capable of killing cells are due to nuclear interactions, while this fraction reduces to $2 \%$ in the BP region [4]. A radiobiological model (LEM [7]) was used to estimate cell survival probability. The expected contribution of target fragments is calculated assuming that in water about $1 \%$ of primary protons undergo nuclear inelastic interactions per traversed $\mathrm{cm}$. Even though both the contributions of ionization and fragmentation increase when approaching the Bragg peak, at that position the biological effect is mainly due to ionization events. On the contrary, in the entrance channel the predicted survival is high, and therefore a significant role might be played by low-energy target fragments.

The lack of information about the impact of target fragmentation on the RBE in CPT is due on the one hand to the difficult determination of the RBE of fragments with biological experiments and in the other hand to the lack of cross section data for the production of heavy recoils after proton irradiation in the energy range of interest. From the above consideration it appears that in 
order to address the problem of possible increased effectiveness of protons in the entrance channel, target fragmentation should be investigated in the energy range 100-250 MeV.

In the energy range of therapeutic application (50-250 MeV for protons and 50-400 MeV/u for carbon ions) the fragmentation process has not been completely covered by experimental measurements and it is necessary to rely upon simulation models. These suffer from many uncertainties since there is no exact calculable theory of these nuclear processes. In recent years some experiments have been dedicated to the study of projectile fragmentation of ${ }^{12} \mathrm{C}$ ions, however this program was carried out only for a few energies [5, 6]. On the other hand the process of target fragmentation, which is the only relevant process of this kind in proton therapy, so far has been almost completely neglected. Actually there are some measurements of light fragment $(\mathrm{A}<3)$ production, but there is a total lack data for larger A values.

\section{The FOOT experiment}

The main experimental difficulty in the measure of the target fragmentation induced by proton beams is due to the short ranges of produced fragments that have low probability to escape the target. In fact their range is confined to tens of microns and even a very thin solid target can badly spoil the fragment energy measurement. A possible workaround can be envisaged in the use of gases target but an inverse kinematic approach can be pursued, studying the fragmentation of different ions beams $\left(\mathrm{C}, \mathrm{O}, \mathrm{Ca}\right.$, etc.) onto hydrogen enriched target, such as $\mathrm{CH}_{2}$, as already adopted in ref.[8, 5]. Secondary fragments will have boosted energy and longer range, making detection easier. The authors of ref. [5] have already shown that the cross section on $\mathrm{H}$ can be extracted by subtraction from the coupled data obtained using both $\mathrm{CH}_{2}$ and pure $\mathrm{C}$ target.

The final goal of the experiment would be to measure the heavy fragment $(Z>3)$ cross section with maximum uncertainty of 5\% and the fragment energy spectrum (in the "patient" reference frame) with an energy resolution of the order of 1-2 MeV/u, in order to contribute to a better radiobiological characterization of protons. The charge and isotopic identification ( at the level of 2-3\% and 5\% respectively) are also the main ingredients of this measurement. In spite that fragmentation nuclear models embedded in simulation software are not reliable to produce sound radiobiological outcome, Monte Carlo simulation can be safely used as guide for the detector design. An example of the prediction from Monte Carlo calculation is given in Fig. 1, where the angular distribution of different fragments produced by a $200 \mathrm{MeV} / \mathrm{u}{ }^{16} \mathrm{O}$ beam impinging on a $\mathrm{CH}_{2}$ target is shown as resulting from the FLUKA code [9]. The typical kinematics of the fragmentation in this energy range is clearly shown: the heavier fragment $(Z \geq 2)$ are forward peaked within an angular cone $\simeq 10^{0}$ around the beam and with a kinetic energy per nucleon peaked around the corresponding value of the beam, while the light fragments have wider angular and kinetic energy distribution. Due to the particular interest of the heavier fragment the experiment is focused on the detection of these higher $\mathrm{Z}$ fragments in a narrow cone $\left(\simeq 10^{0}\right)$ around the beam.

Furthermore, analyzing data in terms of direct kinematics, one can also extend and complete the measurements of projectile fragmentation cross sections induced by $\mathrm{C}$ and $\mathrm{O}$ beams. Such measurements are needed to improve the projectile fragmentation description of these beams in ion therapy and their specific Treatment Planning Systems. 

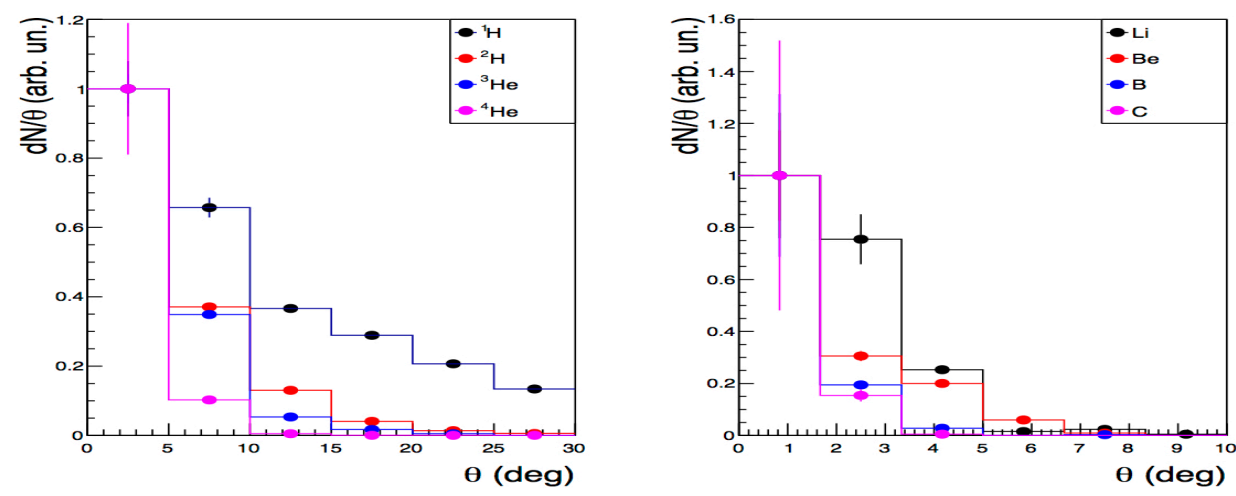

Figure 1: MC calculation [9] of the angular distribution of different fragments produced by a $200 \mathrm{MeV} / \mathrm{u}$ ${ }^{16} \mathrm{O}$ beam impinging on a $\mathrm{CH}_{2}$ target.

The measurements performed with the proposed experiment could be also interesting for other applications, like radioprotection in space. NASA and other space agencies have started since several years the study of the risk assessment for astronauts in view of long duration space missions, such for instance the travel to Mars [10]. Beyond many important risks of other nature, both galactic cosmic rays and particles from the almost unpredictable Solar Particle Events have to be considered. The design and optimization of spacecraft shielding requires a detailed knowledge of fragmentation processes.

\section{The FOOT detector}

The design of the detector is driven mainly by the radiobiology outcome requests. In particular the adoption of the inverse kinematics approach asks not only for a few $\%$ level accuracy on the energy and of momenta of the produced fragments, but also for a resolution on the emission angle with respect to the beam of the order of few mrad. To achieve such an angular resolution, both the beam particles direction before the target and the fragment emission angle after the target must be tracked with an angular accuracy at the mrad level. Furthermore the multiple scattering angle of beam+fragment couple inside the target must be kept as well below the mrad. This sets a severe limit on the allowed thickness of the target (of the order of $1-2 \mathrm{~mm}$ ) and limits accordingly the probability of the fragmentation events to order of $10^{-3}$.

The driving criterion of the FOOT detector design is the need for a robust charge and isotopic identification of the produced fragments. According to this the setup measures the following quantities of the fragments produced: momentum, kinetic energy, Time Of Flight (TOF). The fragment $\frac{d E}{d x}$ is measured through the $\Delta E$ released in a thin slab of material. Using these informations the charge can be identified by the crossing of the $\delta E$ measurement with TOF or kinetic energy, while the mass can be extracted by means of the three kinematic constraints:

$$
p=m c \beta \gamma \quad E_{k i n}=m c^{2}(\gamma-1) \quad E_{k i n}=\sqrt{p^{2} c^{2}-m^{2} c^{4}}-m c^{2}
$$

where $\beta=\frac{v}{c}$ and $\gamma=\frac{1}{\sqrt{1-\beta^{2}}}$ are derived by the TOF of the fragment.

The fragmentation contribution due to detector material must be kept as low as possible and eventually subtracted. An easy detector portability is an absolute need to exploit the availability 
of the needed beams at the therapeutic centre like the Centro Nazionale di Adroterapia Oncologica in Pavia, Italy(CNAO) or Heidelberg Ion Therapy Center, Germany (HIT). As a consequence the overall size of the detector should be in the 1.5-2 meters range.

Even with this redundancy on the mass measurement, the needed fragment identification asks for a detector with the following performances: i) relative resolution on the momentum $\sigma(p) / p$ at level of 3\%; ii) TOF resolution of the order of $100 \mathrm{ps}$; iii) relative resolution on the kinetic energy measurement $\sigma\left(E_{k i n}\right) /\left(E_{K i n}\right)$ at level of $2 \%$; iv) relative resolution of the $\Delta E$ measurement $\sigma(\Delta E) /(\Delta E)$ at level of $2 \%$;

The detector design, following the beam direction, can be divided in three different regions: the upstream/target region, the magnetic tracking region and the calorimeter region. A schematic view of the detector is shown in fig 2

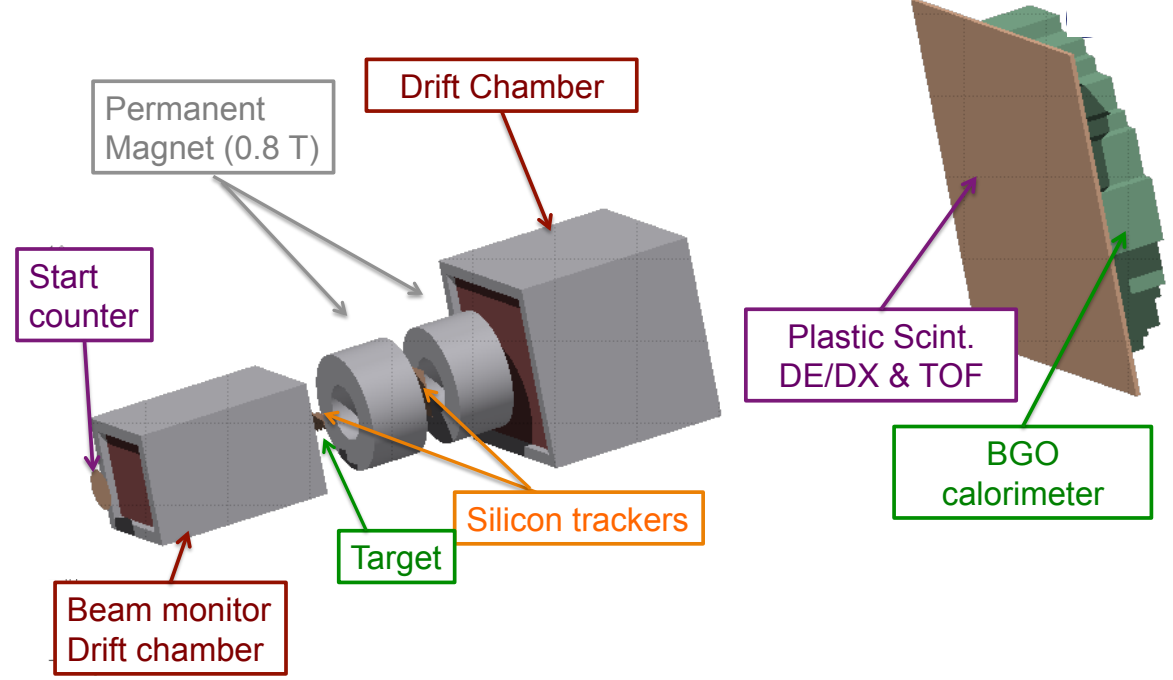

Figure 2: Schematic view of the FOOT apparatus.

- The upstream/target region. Here the beam crosses a thin plastic scintillator counter $(250 \mu \mathrm{m})$ that provides trigger information and the start of the TOF. Then a drift chamber acts as beam monitor and tracks the direction and the position of the beam. The target is the last element of this region

- The magnetic region. After the production in the target the fragments enter in a magnetic region. The magnetic field is provided by two permanent dipole magnets (Halbac geometry). Before the first magnet, between the magnet and after the second magnet, the fragment direction is measured respectively by two silicon pixel trackers and a drift chamber to extract the fragment momentum 
- The calorimeter region. After the magnetic region the fragments travel 1 meter to reach a $\Delta E$ and TOF detector made of two orthogonal planes of $3 \mathrm{~mm}$ thick, $40 \mathrm{~cm}$ long plastic scintillator rods. Finally the fragments kinetic energy is measured in a $10 \mathrm{~cm}$ thick BGO crystal calorimeter. The $20 \mathrm{~cm}$ radius of the calorimeter is dictated by the angular aperture of the heavy fragments.

The multiple measurements of the mass allows to keep under control the effect of the neutron punch through in the calorimeter, that at this energy is present in a sizeable fraction of the events.

\section{Emulsion chambers setup}

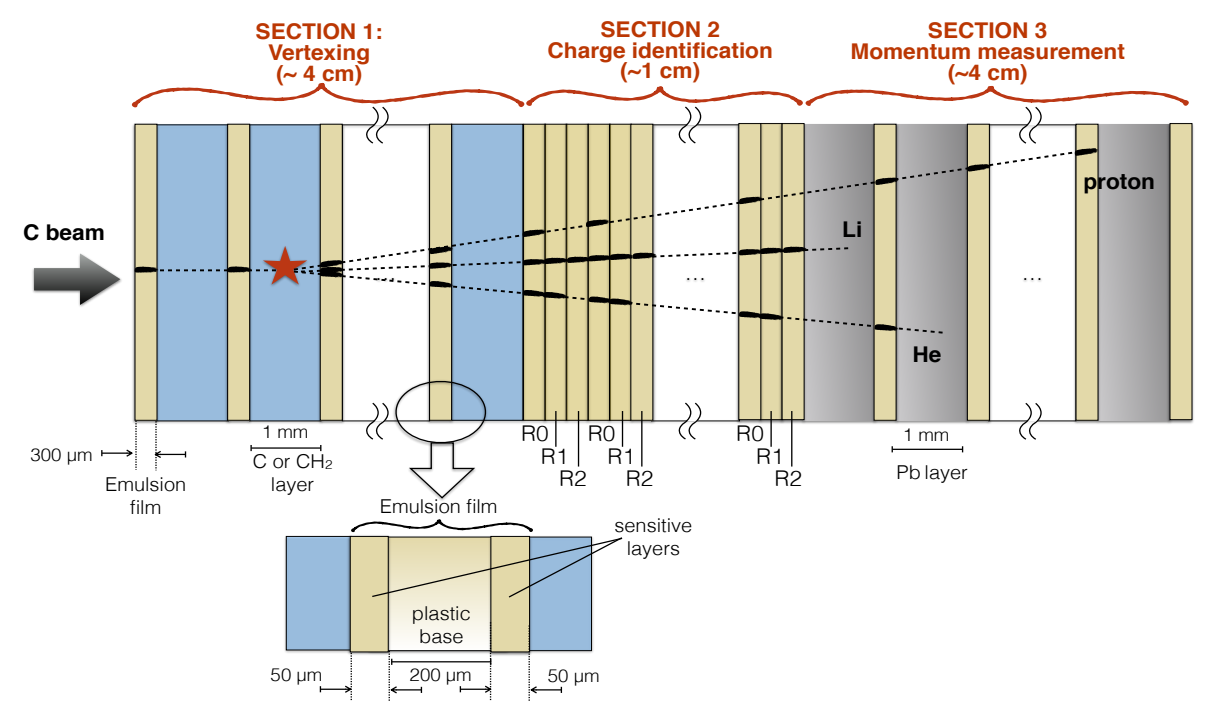

Figure 3: Scheme of the emulsion spectrometer.

The emulsion spectrometer supplies complementary measurements for low $\mathrm{Z}(\mathrm{Z} \leq 3)$ particles and for large angle fragments $[11,12]$ with respect to the electronic detector previously described. The emulsion detector allows to extend the fragments maximum angular acceptance from 10 degrees up to about 70 degrees with respect the beam axis. The emulsion consists of $\mathrm{AgBr}$ crystal scattered in a gelatine binder. The crystals have a diameter of $0.2 \mu \mathrm{m}$ and are sensitive to Minimum Ionizing Particles (MIPs). The trajectory of a MIP is recorded by a series of sensitised $\mathrm{AgBr}$ crystals along its path acting as latent image centres. A chemical process, known as development, enhances latent images, inducing the growth of silver clusters (grains) with a diameter of $0.6 \mu \mathrm{m}$ which can be seen with an optical microscope. The density of grains is proportional to the charge particle ionization within the dynamical range. Besides the sub-micrometric space resolution, another important features of these detectors is that they allow passive and active detector to be integrated in a very compact set-up, so to make the reconstruction of the interactions inside the target possible.

The emulsion spectrometer, based on the concept of Emulsion Cloud Chamber (ECC), will be designed with passive material as carbon target alternated to nuclear emulsions films, acting as both high-resolution tracking devices and ionization detectors. It is planned to be made of three 
sections (Fig. 3): vertex and tracking detector, ionization detector for charge identification and tracking detector for momentum measurements.

The emulsion chamber must be run inside the FOOT setup after the upstream/region part of the detector, with the start counter and beam monitor used for absolute flux normalization. To avoid local pile-up the emulsion camber will be exposed to the beam with a remotely controlled movement system during the data taking.

\section{Conclusion and future schedule}

As stated before the issue of the proton RBE (and of the target fragmentation) is under the spot in the Particle Therapy community, and the FOOT collaboration is designing a detector to measure both target fragmentation in proton therapy than projectile fragmentation in carbon therapy. The R\&D for experiment during 2017 has been approved and funded by INFN, with contribution by Centro Fermi Institute. Final approval for the 2018-2021 period expected in june 2017.

The data taking will take place using $\mathrm{C}, \mathrm{O}(\mathrm{N})$ beams in the 100-350 MeV/u energy range. Further important features of the experimental site could be the possibility to mount and calibrate the experimental setup before data taking for long time (1-2 week), beam time availability in the week range. The preferred site is the experimental hall of the CNAO center in Pavia, Italy and a possible data for the first data taking is late 2018 for the emulsion setup, while late 2019 is the target data taking data for the complete setup

\section{References}

[1] M. Jermann, Int. J. Part. Ther. 2 (2015).

[2] M. Durante and J.S. Loeffler JS ,Nature Rev. Clin. Oncol. 7 (2010).

[3] J.T. Tang JT et al., Br J Cancer 76 (1997).

[4] F. Tommasino and M. Durante, Cancers 12 (2015).

[5] J. Dudouet et al., Phys. Rev. C 88 no. 2 (2013) 064615.

[6] M. Toppi et al., Phys. Rev. C 93 (2016) 064601.

[7] M. Scholz, A.M. Kellerer, W. Kraft-Weyrather, G. Kraft, Radiat. Environ. Biophys. 36 (1997) 59.

[8] W.R. Webber et al. , Phys. Rev. C 41 no. 2 (1990).

[9] A. Ferrari et al., CERN 2005-10, INFN/TC_05/11, SLAC-R-773L (2005); T.T. Böhlen et al. Nuclear Data Sheets 120 (2014) 211.

[10] M. Durante and F.A. Cucinotta "Physical basis of radiation protection in space travel" Rev. Mod. Phys. 83 (2011) 1245.

[11] G. De Lellis et al., Nuclear Physics A 853 (2011) 124Đ134

[12] G. De Lellis et al., JINST 2 (2007) P06004. 